\title{
O papel da enfermagem frente às mudanças sexuais no período do climatério
}

\author{
The role of nursing in the face of sexual changes in the climacteric period \\ El papel de la enfermería ante los cambios sexuales en el período climatérico
}

\section{Resumo}

Objetivo: Este estudo surgiu devido a necessidade de avaliar o conhecimento sobre sexualidade no climatério entre os enfermeiros da Estratégia Saúde da Família. Método: Pesquisa de campo, com abordagem quantitativa, de cunho descritivo e de corte transversal. A coleta foi realizada no período de Junho à Julho de 2015. Resultados: Para a avaliação do questionário foram consultados 13 profissionais para avaliar o questionário que trata sobre sexualidade humana. Em termos gerais, apenas um dos experts consideraram o questionário razoável. Sendo desta forma o questionário aprovado pela etapa dos experts. Foi aplicado ainda o coeficiente de confiabilidade de alfa de Cronbach obtendo resultado igual a 0,86 . O que indica sua confiabilidade nas respostas dos experts. Considerações finais: Existe a necessidade de transversalidade do cuidado de enfermagem à mulher no climatério desde o tratar do cuidado do corpo com propriedade e intimidade com o mesmo, seus aspectos mais elementares do cuidado com a higiene corporal até aqueles tecnicamente mais complexos, como identificar inadequações e disfunções sexuais prescrevendo os cuidados de enfermagem.

Palavras-chave: Sexualidade; Climatério; Atenção primária; Enfermagem.

\begin{abstract}
Objective: This study arose out of the need to assess knowledge about sexuality in menopause among nurses in the Family Health Strategy. Method: Field research, with a quantitative approach, descriptive and cross-sectional. Data collection was carried out from June to July 2015. Results: For the evaluation of the questionnaire, 13 professionals were consulted to evaluate the questionnaire that deals with human sexuality. Overall, only one of the experts considered the questionnaire to be reasonable. Thus, the questionnaire was approved by the experts' stage. Cronbach's alpha reliability coefficient was also applied, obtaining a result equal to 0.86 . Which indicates its reliability in the experts' answers. Final considerations: There is a need for transversality of nursing care to women in menopause, from dealing with the care of the body with propriety and intimacy with it, its most elementary aspects of body hygiene care, to those technically more complex, such as identifying inadequacies and sexual dysfunctions prescribing nursing care.
\end{abstract}

Keywords: Sexuality; Climacteric; Primary attention; Nursing.

\section{Resumen}

Objetivo: Este estudio surge de la necesidad de evaluar el conocimiento sobre la sexualidad en la menopausia entre enfermeras en la Estrategia Salud de la Familia. Método: Investigación de campo, con enfoque cuantitativo, descriptivo y transversal. La recolección de datos se realizó de junio a julio de 2015. Resultados: Para la evaluación del cuestionario 
se consultó a 13 profesionales para evaluar el cuestionario que trata sobre la sexualidad humana. En general, solo uno de los expertos consideró que el cuestionario era razonable. Así, el cuestionario fue aprobado por la etapa de expertos. También se aplicó el coeficiente de confiabilidad alfa de Cronbach, obteniendo un resultado igual a 0.86. Lo que indica su confiabilidad en las respuestas de los expertos. Consideraciones finales: Existe una necesidad de transversalidad de los cuidados de enfermería a la mujer en la menopausia, desde tratar el cuidado del cuerpo con decoro e intimidad con él, sus aspectos más elementales del cuidado de la higiene corporal, hasta aquellos técnicamente más complejos, como identificar Insuficiencias y disfunciones sexuales que prescriben cuidados de enfermería.

Palabras clave: Sexualidad; Climatérico; Atención primaria; Enfermería.

\section{Introdução}

O climatério é definido como uma fase biológica da vida e não um processo patológico que compreende a transição entre o período reprodutivo e o não reprodutivo da vida da mulher (Valença et al., 2010). A função sexual não só sofre alterações biológicas com a idade, mas também pelas questões emocionais, sociais e culturais que interferem, significativamente, na intensidade e qualidade do desejo e da resposta sexual (Lima Gonçalves et al., 2013; Silva, Dias \& Oliveira, 2019). O aumento da expectativa de vida da população tem se refletido na conformação da população brasileira (De Souza et al., 2021). Em nosso país, as mulheres representam a maioria da população idosa.

O desconhecimento e os tabus que delineiam a sexualidade durante o climatério acentuam as mudanças que acontecem no corpo e nas relações sexuais nesse período da vida, atribuindo, de forma equivocada, a diminuição do desejo a menopausa (Alves, 2020). A maior parte das barreiras não são propriamente sexuais, mas sim problemas e dificuldades que se refletem na vida sexual, como as normas e dinâmicas de gênero (Mendes Pinto Neto et al., 2012).

No entanto, a sexualidade no climatério deve ser compreendida também a partir de fenômenos não lineares, considerando a importância da associação entre a vivência individual e a dinâmica da intimidade emocional e da satisfação no relacionamento (Brasil, 2013).

A sexualidade é uma dimensão fundamental de todas as etapas da vida de mulheres e homens, presente desde o nascimento até a morte (Fettermann et al., 2021). Por essa razão, é necessário valorizar, promover e incentivar o autoconhecimento, que implica buscar conhecer a si mesma, seus valores, seu modo de ver e viver a sexualidade e a relação com o outro. Cruz da Silva et al., (2013), nos trazem informações que mostram que mesmo a sexualidade sendo estudada desde o século anterior, o enfermeiro tem dificuldade de trabalhar o tema devido a falta de disciplinas na graduação que abordem as interfaces da sexualidade de modo a fornecer conhecimento específico ao profissional.

Outros estudos nesta área também focaram na avaliação do conhecimento do acadêmico de enfermagem sobre sexualidade e as grades curriculares da graduação de suas universidades. Da mesma forma, concluíram que existe a necessidade de fortalecer a graduação com disciplina específica e que seja tratado o tema também de forma transversal à outras disciplinas, com o intuito de preparar melhor o profissional (Gd et al., 2013; Sodson Da \& Silva, 2009; Zampieri et al., 2009).

Estas informações sobre sexualidade, climatério e sobre o papel do enfermeiro frente a este cenário, visto que o mesmo trás na base da sua profissão a essência do cuidar, e com isso a preocupação com as questões do desenvolvimento da habilidade e competência profissional para que as ações sejam disparadas pelo saber científico. Tratar de assuntos tão delicados e relevantes exigem, do profissional o conhecimento das especificidades, que neste caso, trata-se de áreas de conhecimento muito específicas, onde os estudos supracitados fomentam e apontam dificuldades na formação profissional e consequentemente na forma de desempenhar a prática assistencial do cuidar.

O que nos remete a alguns questionamentos: O profissional enfermeiro entende como a mulher estaria vivenciando as alterações sexuais no período do climatério? O enfermeiro sabe quais alterações sexuais são evidenciadas pela mulher nesse momento da vida, e como se relacionam com sua sexualidade? Compreende como a mulher expressa as questões relacionadas à sexualidade? 
Diante de tantos questionamentos, surge a necessidade deste estudo a fim de saber sobre o conhecimento do enfermeiro da Atenção primária sobre sexualidade da mulher no climatério e suas interfaces. E com isso, objetivou-se avaliar o conhecimento sobre sexualidade no climatério entre os enfermeiros da Estratégia Saúde da Família.

\section{Metodologia \\ Tipo de estudo}

Trata-se de uma pesquisa de campo, com abordagem quantitativa, de cunho descritivo e de corte transversal.

A abordagem é quantitativa uma vez que suas variáveis referem-se a propriedades que mantêm a mesma natureza em toda sua extensão ou dimensão, podendo ser manifestados em termos numéricos (Medronho, 2009).

Local do Estudo: A pesquisa foi realizada no município de Recife-PE possui população estimada de 1.599 .514 habitantes e densidade demográfica de 7.039,64 hab/km². (IBGE). O município tem 250 Equipes de Saúde da Família.

\section{População e amostra}

A população em estudo é composta pelos 250 enfermeiros que atuam nas Equipes de Saúde da Família (ESF) do município de Recife e determinação de amostra por conveniência.

\section{Coleta de dados}

Estudo transversal com enfermeiros que trabalham na atenção básica no município de Recife-PE por meio de questionário auto-preenchido.

A coleta foi realizada no período de Junho à Julho de 2015, após explanação do objeto de pesquisa por uma mestranda (pesquisadora responsável pelo projeto).

Compreendeu as seguintes etapas:

a) Apresentação da pesquisa;

b) Leitura do Termo de Consentimento Livre e Esclarecido (TCLE) e instruções para o preenchimento;

c) Aplicação do instrumento entre as participantes;

A coleta de dados aconteceu nas reuniões de matriciamento da Atenção primária. Após a explicação dos procedimentos e objetivos da pesquisa e assinatura do Termo de Consentimento Livre e Esclarecido (TCLE), o questionário foi entregue e iniciou-se com o preenchimento dos dados, ele mesmo respondeu todas as partes, (visto que esses instrumentos foram criados para serem auto-aplicáveis). Os participantes depositaram respectivamente em duas caixas os questionários preenchidos e na outra os TCLE's para garantir o sigilo da resposta.

\section{Organização e análise dos dados}

Foram apresentadas as estatísticas descritivas por meio de distribuição de frequências para as variáveis categóricas e médias com suas respectivas variações para as variáveis quantitativas.

Os escores de cada domínio foram calculados pela média da pontuação (escala likert) das questões relacionadas a cada um dos domínios:

1. Conhecimento sobre sexualidade Geral;

2. Conhecimento sobre Política de Atenção à mulher no climatério,

3. Conhecimento sobre Sexualidade no climatério

4. Conhecimento sobre o caderno $n^{\circ} 26$. 
Foi aplicado o teste de Shapiro-Wilks para a comprovação de normalidade na distribuição dos escores em cada um dos domínios. Na comparação dos escores médios em cada um dos domínios e as características sociodemográficas e relacionadas à formação profissional foi aplicada a ANOVA com pós-teste de Bonferroni. A significância estatística adotada no estudo foi de $5 \%(\mathrm{p}<0,05)$. O software utilizado na análise foi o STATA versão 12.0.

\section{Aspectos éticos e legais}

O Termo de Consentimento Livre e Esclarecido foi construído a partir do modelo orientado pela Plataforma Brasil e foi aplicado aos sujeitos da pesquisa assegurando a observação dos princípios éticos descritos na Resolução n ${ }^{\circ}$ 196/96 do CNS, que atende às exigências da Resolução 466/12, que trata da pesquisa envolvendo seres humanos.

Após aprovação do Comitê de Ética e Pesquisa com Seres Humanos da Universidade de Pernambuco, recebido em 01 de Dezembro de 2014, CAAE: 39337714.1.0000.5207. Foi emitida a carta de anuência pela Prefeitura da Cidade do Recife para que a pesquisa fosse iniciada.

\section{Riscos e benefícios}

Os riscos foram mínimos, uma vez que os dados foram coletados mediante o questionário auto-aplicável, com uso apenas de caneta e papel.

Caso o entrevistado viesse a sentir-se desconfortável ou constrangido, a pesquisa seria interrompida imediatamente. Para o serviço de saúde espera-se que os resultados da pesquisa subsidiam uma descrição detalhada das características do saber do enfermeiro com relação à sexualidade no climatério e o impacto que a sexualidade traz o pode influenciar sua qualidade de vida, o que possibilita um melhor conhecimento acerca da paciente/cliente/usuária e de sua adaptação à condição de estar vivendo uma fase específica da vida.

\section{Resultados}

\section{Validação do questionário}

Para a avaliação do questionário foram consultados 13 profissionais para avaliar o questionário que trata sobre sexualidade humana. Em termos gerais, apenas um dos experts consideraram o questionário razoável. Sendo desta forma o questionário aprovado pela etapa dos experts.

Foi aplicado ainda o coeficiente de confiabilidade de alfa de Cronbach obtendo resultado igual a 0,86 . O que indica sua confiabilidade nas respostas dos experts. O Quadro 1 mostra que 92,2\% dos experts aprovaram o questionário sem ressalvas.

Quadro 1. Avaliação geral do questionário pelos experts. Recife, Pernambuco, Brasil.

\begin{tabular}{|l|l|}
\hline \multicolumn{2}{|c|}{ Avaliação Geral } \\
\hline Razoável & $1(7,8 \%)$ \\
\hline Bom & $6(46,1 \%)$ \\
\hline Excelente & $6(46,1 \%)$ \\
\hline
\end{tabular}

Fonte: Pontes, et al (2021).

\section{Aplicação do questionário}

\section{Caracterização do perfil sociodemográfico (SD) das mulheres avaliadas}

Foram pesquisados 250 profissionais de enfermagem que atuam na estratégia da saúde da família, dos quais 90,3\% eram do sexo feminino. A média de idade dos pesquisados foi de 40 anos, idade mínima de 21 e máxima de 80 anos. Estratificados em faixas etárias, $45,3 \%$ dos enfermeiros tinham idade entre 30 e 39 anos e 29,4\% de 40 a 49 anos. Segundo o estado civil, 
aproximadamente, $60 \%$ dos pesquisados são casados, sendo $30 \%$ solteiros e 10,1\% divorciados ou viúvos. Quanto à condição de ter filhos, $71,7 \%$ têm filhos, desses $87 \%$ têm um ou dois filhos. A religião da maioria dos pesquisados é a católica, sendo 21,1\% evangélicos e 12,2\% espíritas, cerca de 10\% dos entrevistados declararam não ter religião (Quadro 1).

Quanto ao tempo de formação em enfermagem, 16,2\% tem menos de 5 anos de formação, enquanto que $25,5 \%$ tem de 5 a 10 anos e a maioria tem mais de 10 anos de formação. A maioria dos enfermeiros se graduou em uma instituição de ensino superior pública (72,5\%), e apenas 9 dos 250 pesquisados não tinham pós-graduação (3,7\%). Quanto à formação em sexualidade humana, 38,1\% afirmaram ter tido capacitação nessa área. O tempo de atuação profissional em estratégia da saúde da família da maioria dos enfermeiros é de mais de 10 anos (66,8\%) (Quadro 1).

Ainda a respeito da formação dos enfermeiros, quando questionados sobre como poderia ser abordado o tema da sexualidade na graduação e pós-graduação, na graduação, 75,2\% dos pesquisados opinaram que esse tema fosse abordado em uma disciplina, enquanto que 15,3\% em treinamentos, 7,8\% a abordagem seria em seminários e 1,7\% em congressos. Já na pósgraduação, 45,3\% dos enfermeiros opinaram que o assunto sobre sexualidade deveria ser estudado em treinamentos, enquanto que $1 / 3$ deles poderia ser estudado em oficinas, $10,7 \%$ em seminários, 7,8\% em palestras e 2,9\% em congressos.

$\mathrm{Na}$ avaliação do nível de conhecimento dos enfermeiros sobre sexualidade, o questionário foi dividido em 4 domínios: conhecimento sobre sexualidade geral, sobre política de atenção à mulher no climatério, sexualidade no climatério e conhecimento sobre o caderno que fala sobre atenção à saúde sexual e reprodutiva. Os escores médios em cada domínio são pontuação que variam entre 1 e 5, onde, quanto maior o escore maior o nível de conhecimento do domínio estudado.

Segundo o gráfico 2, observa-se que o domínio sobre sexualidade geral apresentou a maior média entre os domínios, com uma média de 3,61 em uma pontuação máxima de 5 pontos, o que representa um nível de conhecimento médio de $72,2 \%$, em percentuais. Em relação ao domínio sobre o conhecimento das políticas de atenção à mulher no climatério, o escore médio foi de 2,56, o que representa um nível de conhecimento de 51,2\%. Quanto ao conhecimento relacionado à sexualidade no climatério, o escore médio foi de 2,67 (nível de conhecimento de 53,4\%). Já quando relacionado ao caderno que fala sobre atenção à saúde sexual e reprodutiva, o escore médio foi de 3,37, o que representa em uma escala de 0 a 100 , um nível de conhecimento de $67,4 \%$. 
Gráfico 1. Distribuição dos escores médios dos enfermeiros sobre os domínios relacionados à sexualidade geral, sobre política de atenção à mulher no climatério, sexualidade no climatério e conhecimento sobre o caderno que fala sobre atenção à saúde sexual e reprodutiva. Recife, Pernambuco, Brasil.

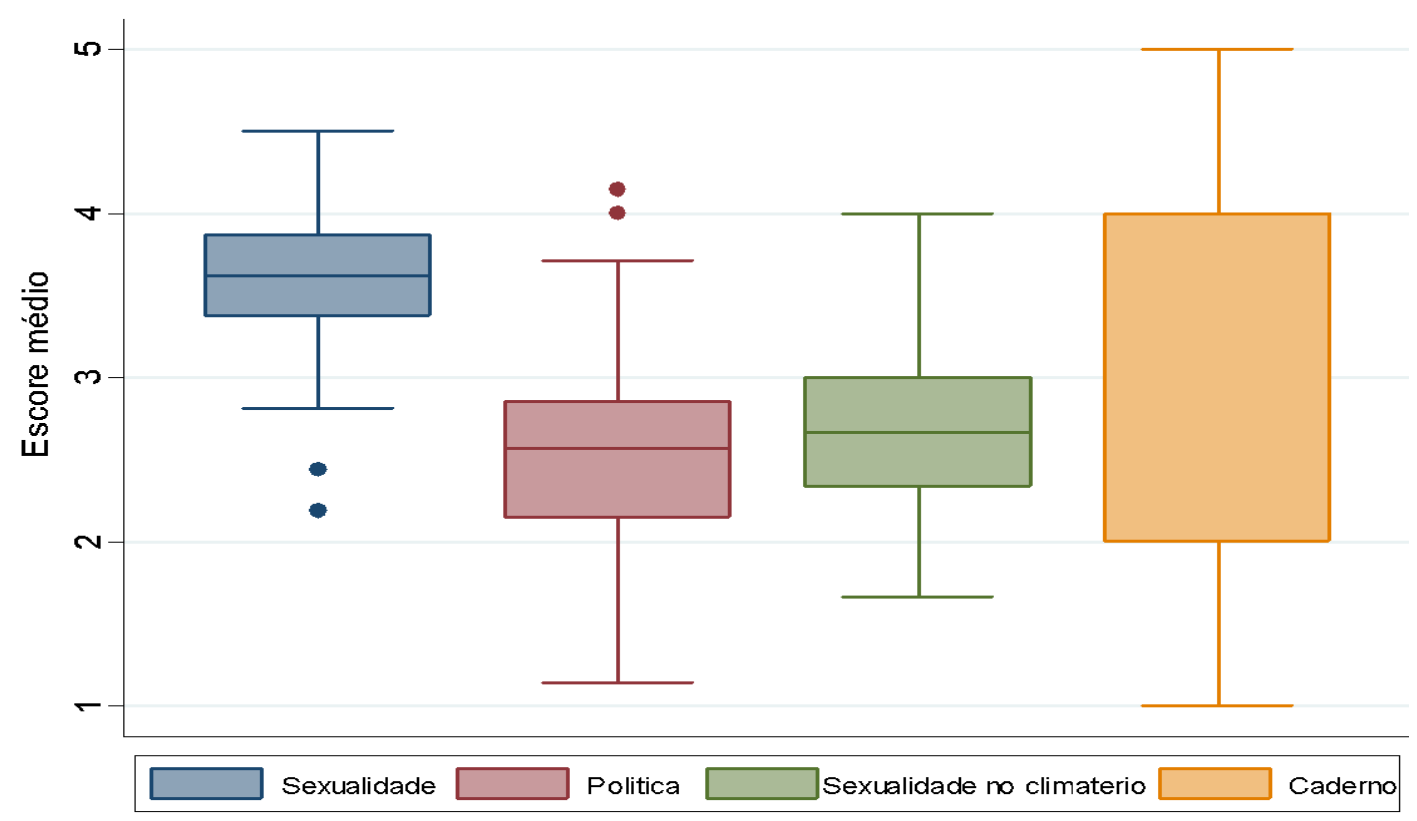

Fonte: Pontes, et al (2021).

De acordo com a Quadro 2, observa-se que quando comparadas as médias dos escores sobre o conhecimento sobre sexualidade geral, houve diferença estatisticamente significativa em relação à faixa etária, onde os profissionais com idade acima dos 50 anos apresentaram os maiores escores (maior nível de conhecimento) quando comparados aos que tinha idade abaixo dos $30 \operatorname{anos}(\mathrm{p}=0,004)$.

Quadro 2. Médias dos escores sobre o conhecimento sobre sexualidade geral. Recife, Pernambuco, Brasil.

\begin{tabular}{|l|l|}
\hline Domínios & Média $\pm \mathbf{d p}$ \\
\hline Sexualidade geral & $3,61 \pm 0,37$ \\
\hline Política & $2,56 \pm 0,52$ \\
\hline Sexualidade no climatério & $2,67 \pm 0,48$ \\
\hline Caderno $^{\circ} 26$ & $3,37 \pm 1,31$ \\
\hline
\end{tabular}

Fonte: Pontes, et al (2021).

Não houve diferença estatisticamente significativa quando comparadas as faixas etárias de 30 a 39,40 a 49 e maior de 50 anos. Em relação a religião a significância estatística foi limítrofe(p = 0,052), onde os profissionais que se declararam sem religião ou que eram espíritas obtiveram as maiores médias do escore quando comparados aos que se declararam católicos ou evangélicos, porém as diferenças entre cada um dos grupos não foi estatisticamente significante $(\mathrm{p}>0,05)$.

Quanto ao conhecimento sobre a política de atenção à mulher no climatério, a associação foi estatisticamente significativa segundo o estado civil ( $p=0,023)$, a condição de ter filhos $(p=0,033)$ e segundo a religião do pesquisado $(p=0,014)$, no qual os enfermeiros solteiros, com filhos e que se declararam sem religião apresentaram as maiores médias do escore, ou seja, apresentaram um maior nível de conhecimento. 
Quanto ao escore relacionado ao conhecimento da sexualidade no climatério e conhecimento sobre o caderno que fala sobre atenção à saúde sexual e reprodutiva, nenhuma das características sociodemográficas apresentou diferença estatisticamente significativa (Quadro 2).

Gráfico 2. Distribuição da média geral das opiniões dos enfermeiros em cada uma das questões relacionadas à sexualidade geral, sobre política de atenção à mulher no climatério, sexualidade no climatério e conhecimento sobre o caderno que fala sobre atenção à saúde sexual e reprodutiva. Recife, Pernambuco, Brasil.

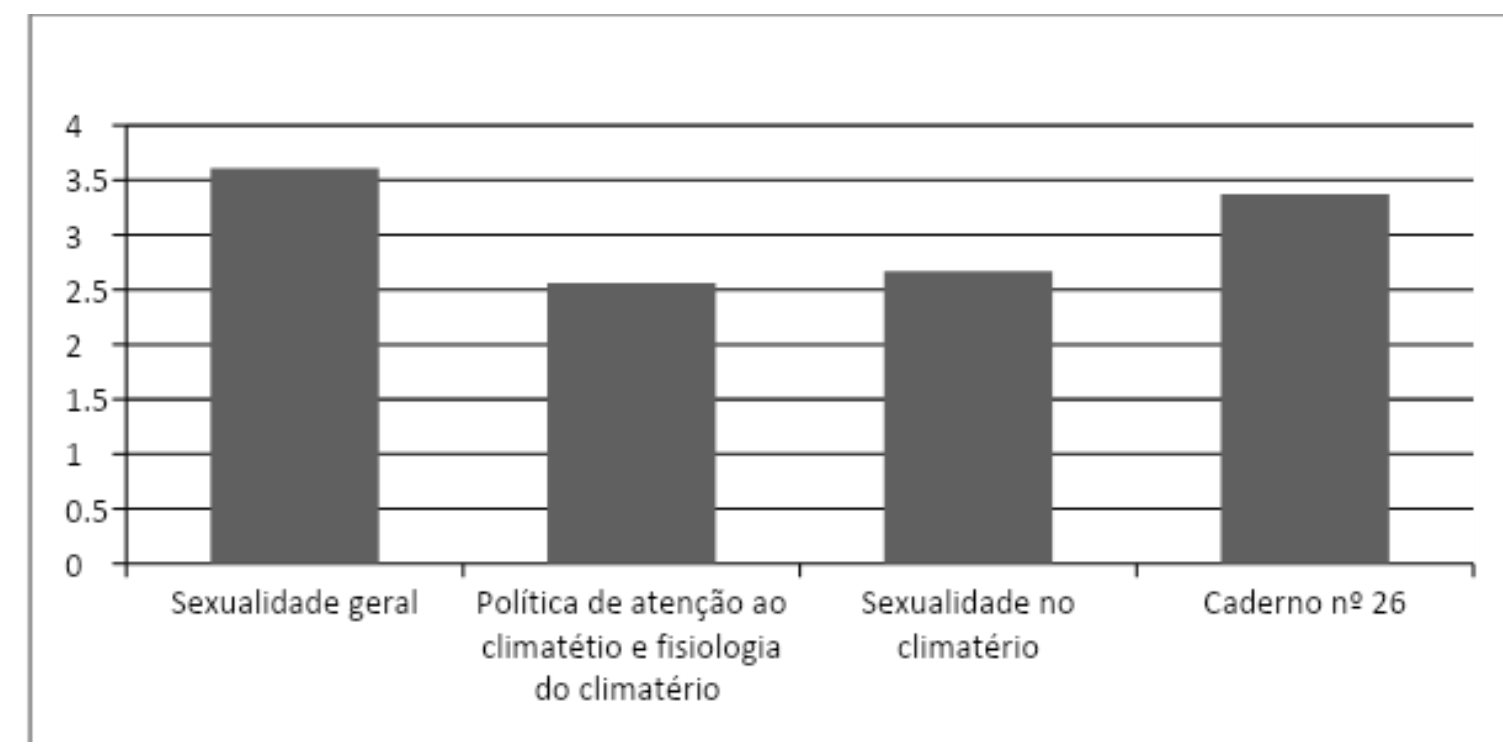

Fonte: Pontes, et al. (2021).

\section{Aplicação do questionário}

Variáveis demográficas, capacitação e experiência dos enfermeiros entrevistados.

Conhecer o perfil e a formação dos profissionais que atuam nas ESFs e cooperar na edificação de ações formativas e educativas, possibilitando a implementação e a efetividade dos princípios da Estratégia. Por isso, é necessário atentar para as características desses profissionais, a fim de propiciar a participação e o comprometimento, que são atitudes fundamentais para o planejamento, desenvolvimento e avaliação das ações (Zanetti et al., 2010).

Observamos predominância do sexo feminino na população estudada (90,3\%) o que também foi percebido de outros estudos que analisaram o perfil de enfermeiros, onde historicamente é tida como uma profissão feminina até pela sua história original. Em Cuiabá - MT em estudo semelhante achou-se o valor de 88,6\% de enfermeiras do sexo feminino. Isso fica bem evidente em outros artigos que buscam o perfil do enfermeiro da Atenção (Fundação Oswaldo Cruz. \& Escorel, 2005).

Com relação à faixa etária dos enfermeiros sujeitos da pesquisa, tivemos 45,3\% com idade entre 31 e 40 anos. O que foi análogo ao do estudo de (Rocha et al., 2009). que teve uma população semelhante em idade e área de atuação. A média de idade encontrada neste estudo (29,4\% estavam entre 40-49 anos Estudo nacional observou uma faixa etária acima dos 30 anos, porém com uma média de 34 anos (Fundação Oswaldo Cruz. \& Escorel, 2005; Machado, 2014). Tal fato deve estar relacionado ao fato que na cidade de Recife a implantação da ESF de deu a mais tempo, segundo este estudo nacional a implantação nos locais estudados tinha uma média de 8 anos, enquanto em recife o primeiro PSF implantado data de Junho de 1997, portanto 18 anos de implantação, perfazendo uma diferença de 10 ano a mais.

Tivemos um predomínio de população com estado civil declarado como casado (60\% dos pesquisados), ficando entre o que (Zanetti et al., 2010; Canesqui \& Spinelli, 2006; Lopes \& Bousquat, 2011) encontraram em sua pesquisa que foi igual a 70,8\% e (Canesqui \& Spinelli, 2006; Lopes \& Bousquat, 2011). Com relação à realização de Pós-graduação se assemelham aos 
de onde aproximadamente 95\% tinham cursando pós-graduação na pesquisa de (Ferrari et al., 2005) realizado em Londrina (Paraná), e diferem dos dados encontrados por (Zanetti et al., 2010) que encontraram 63,3\%; Diniz Machado et al., (2014) com $73,7 \%$. Ressaltando que nos últimos cinco anos as pós-graduações aumentaram muito em nosso estado.

Encontramos 66,8\% dos enfermeiros atuavam na Atenção Básica à Saúde a mais de 10 anos, o que foi superior ao de (Ferrari et al., 2005), onde a maioria dos enfermeiros entrevistados (80,9\%), tinha mais de dois anos de ESF, com uma média de 2,4 anos, Lopes e Bousquat (2011). Reforçando a consolidação da ESF em Recife pelo tempo de implantação, e por ter havido concurso para ESF o que dá estabilidade profissional.

\section{Discussão}

\section{Conhecimentos dos enfermeiros sobre climatério}

Neste estudo, por todas as descrições SD encontradas, pelo tempo de serviço, pela formação profissional, por se tratar de uma população predominantemente feminina e pela idade dos pesquisados, sugere-nos um conhecimento maior sobre a política de atenção à saúde da mulher no climatério, e também sobre os aspectos fisiológicos do climatério. Até porque se trata de um assunto que além de abordado na universidade é uma das políticas mais antigas (Cruz da Silva et al., 2013.).

No entanto, ao relacionarmos a idade ao escore de conhecimento, temos um escore de 2,71 entre os enfermeiros que têm entre 21-29 anos como sendo o mais alto, sugerindo que os mais novos estão com mais conhecimento sobre esse tema. Consequentemente têm menos tempo de formado e os assuntos da universidade são mais facilmente lembrados. Com relação ao gênero, ambos demonstraram igual conhecimento com o assunto, em torno de 50\%. Mostrando que achar que as mulheres entenderiam mais é um mito de gênero. Quanto aos padrões de conhecimento na enfermagem, os componentes estético, ético e pessoal têm em comum a percepção e a intuição.

Os que declararam não casados (ou com união), assim como os que têm filhos tiveram escores superiores. Com relação à religião nos chama atenção que com o escore de 2,83, o mais alto escore encontrado nesta segmentação, encontram-se os que não têm religião.

Com relação ao maior tempo de formado não teve repercussão positiva sobre o conhecimento da política nem da fisiologia (os enfermeiros com menos tempo de formado demonstraram mais informação assim como relacionamos à idade). Em contrapartida, o tempo de experiência profissional na ESF sugere que o desenvolvimento da prática profissional conduz o enfermeiro ao empoderamento, demonstrando mais conhecimento de acordo com o tempo, tendo em vista que o aprendizado não se constrói sozinho, mas com a inter-relação com outras pessoas (Cestari, 2003). Há, contudo, um outro elemento que surge como pré-condição para esses padrões de conhecimento - a experiência (Lopes \& Bousquat, 2011).

Ito et al., (2010) relaciona as mudanças de incentivo social e de políticas de saúde como impulsionadores que podem influenciar no ensino da enfermagem. Estas transformações influenciam as modificações nos Projetos Político-Pedagógicos (PPP) dos cursos de graduação, que entusiasmam a formação profissional do enfermeiro para uma conformação generalista, com competência e habilidades acadêmico-científicas e ético-humanísticas em consonância com os preceitos do SUS (Lavôr, 2010).

Tudo isso faz o profissional ser direcionado às diretrizes do SUS, aproximando-o das políticas de saúde e vendo o usuário de forma integral. O que beneficia as mulheres, devido ao maior conhecimento pelo enfermeiro da política de saúde da mulher (PNAISM) e consequentemente sobre a política de atenção à mulher no climatério.

Com relação ao Manual de Atenção à Mulher no Climatério que tem a última versão em 2008, disponível online e na maioria das ESFs, não justifica o desconhecimento das suas diretrizes, do seu conteúdo. Na média geral de conhecimento sobre o tema, os enfermeiros atingiram o escore de 2,56, que foi a área de menor conhecimento entre as quatro contidas na pesquisa.

(Brasil, 2008). 
De forma geral as bases de dados não fornecem estudos quantitativos para avaliação de conhecimento, encontramos alguns qualitativos, no entanto estes não falam sobre climatério a fim de fazermos a análise comparativa, (LAVÔR, 2010) assim como (Carla et al., 2010) em suas pesquisas qualitativas, descrevem o pouco conhecimento da enfermagem com relação ao climatério, inclusive descrevem que os enfermeiros participantes de suas pesquisas, em sua maioria, confundiram os conceitos de menopausa e climatério.

Principalmente quando sabemos que o climatério é em um momento importante na vida das mulheres onde elas precisam se tornar sujeitos autônomos em relação ao auto-cuidado. Isso exige que os profissionais de saúde estejam capacitados e prontos a agirem de modo holístico com uma abordagem tanto física quanto sócio emocional (Costa \& Miranda, 2010).

Não deixando de lado o importante papel do enfermeiro como multiplicador de informações à equipe e seu papel na educação permanente, que além de política pública é a principal forma de empoderamento dos (as) usuários (as) (e para isto é preciso o conhecimento). Além de neste caso das mulheres que estão no climatério, viverem em uma sociedade onde os mitos e tabus característicos desta fase, são limitadores da qualidade de vida, o que exige mais ainda do profissional por ser formador de opinião.

\section{Conhecimentos dos enfermeiros sobre sexualidade}

A Sexualidade Humana (SH) por si é pouco estudada na enfermagem, realizando buscas na Coordenação de Aperfeiçoamento de Pessoal de Nível Superior (CAPES), Medical Literature Analysis and Retrievel System Online ( MEDLINE), Cochrane Library e Biblioteca Virtual em Saúde (BVS), em uma série histórica de 20 anos temos 3396 artigos publicados. Quando refinamos a busca e retiramos os repetidos, e os que não envolvem a enfermagem restam 438. Destacando que em sua maioria falam de sexualidade relacionado à infecções sexualmente transmissíveis (IST'S), principalmente sobre o vírus da imunodeficiência humana (HIV/Aids), e sobre gravidez na adolescência. Nos últimos cinco anos começaram a surgir publicações na área de diferença de gênero e orientação sexual.

Fato esse que é preocupante porque as publicações são o reflexo dos estudos realizados, e são oportunidade de obtenção de conhecimento recente e validado. A enfermagem precisa ter conhecimento na área para atuar com conhecimento e habilidade, o que permitirá ao profissional ter mais segurança e melhor desempenho. Sem falar que por si a sexualidade já tem entraves devido à cultura e o forte papel das religiões.

$\mathrm{Na}$ atenção primária o enfermeiro desempenha um papel importantíssimo na promoção à saúde, onde a educação em saúde é a base para fazer a educação permanente da equipe e da comunidade. E ainda através da educação popular em saúde, que é uma metodologia Freireana para fortalecer o empoderamento das pessoas desfazendo preconceitos, mitos e tabus.

A reflexão sobre a educação popular em saúde no contexto do educar-cuidar da enfermeira, sob o enfoque crítico e problematizador, abarca os princípios da prática educativo-dialógica baseada na filosofia freireana, sendo empregados na pedagogia da saúde e, com isso, trazendo a oportunidade de transformar a prática da enfermagem. Essa filosofia, aplicada à prática educativa da enfermeira, proporciona a mudança de saberes através da crítica e reflexão, dentro de uma comunidade que não possui conhecimento científico, além de adequar-se ao conhecimento vindo do senso comum. A partir disso, entende-se que a enfermeira deve ter uma ação coletiva associada com o saber popular no espaço educativo em saúde pois, se não agir dessa forma, o conhecimento científico permanecerá anacrônico e desassociado do mundo prático (Aparecida et al., 2007).

Compete à enfermagem, de um maneira geral, abordar de forma assertiva subsídios às usuárias em relação nas suas condutas no processo que visa garantir saúde, assim (re)construindo conceitos, tornando-se o ator principal do seu cuidar que em sexualidade pode fazer toda diferença na forma de compreensão dos fatos e reformulação de conceitos (Cruz da Silva et al., 2013.). 
Entre os enfermeiros que participaram desta pesquisa encontramos o melhor escore entre os quatro pesquisados quando observamos o conhecimento no domínio de sexualidade geral, totalizando 3,61 (72,2\%). E ainda destacamos o conhecimento destes enfermeiros sobre o caderno de Atenção à saúde sexual e reprodutiva (Brasil, 2010), que ficou em segundo lugar geral de maior escore, totalizando $3,37(67,4 \%)$.

Isso sugere que o conhecimento sobre este caderno destinado aos profissionais da atenção primária influenciou positivamente os profissionais a adquirir maior conhecimento na área, visto que ele aborda principalmente a sexualidade geral, em sua base de conceitos, sinais e sintomas de inadequações e disfunções sexuais, e ainda sugere algumas condutas com base na educação e orientação individual e em grupo, e encaminhamento quando necessário.

O bom desempenho dos enfermeiros nestes dois escores, somados às informações do Gráfico 1, onde os enfermeiros apontam que seria importante ter uma disciplina na faculdade que abordasse sexualidade humana (75,2\%), e que após o término da graduação 33,3\% manifestam vontade de participar de oficinas e 45,3\% de participarem de treinamentos específicos em SH.

$\mathrm{O}$ que demonstra o interesse na busca do saber científico pelo enfermeiro. E fica claro que eles preferem que essas informações sejam repassadas em um modelo didático-pedagógico onde eles tenham mais oportunidade de realizar troca, construção coletiva deste momento. Visto que em oficinas e treinamentos o grupo é menor e não necessariamente temos locutor e espectadores, o que dificultaria perguntas e respostas.

Sendo a sexualidade um dos pilares da qualidade de vida segundo a Organização Mundial da Saúde (OMS), e sabendo que a sexualidade permeia todas as pessoas em todas as fases da vida, é importantíssimo que os profissionais sejam incentivados nesta busca do conhecimento para que isso permeia seu "EU" e consequentemente se livre dos preconceitos e tabus. Isso interfere diretamente em seu modo de ser e de interagir, consequentemente interfere na forma de ver e conviver com pessoas, seja nas relações sociais ou de trabalho. Na ESF, pessoas de diferentes crenças, gênero e orientações, precisam de atendimento, aconselhamento, orientação.

Além deste incentivo, precisamos também investir nas pesquisas científicas que podem e devem ser realizadas na ESF pela própria equipe, visto que as características macro e micro-culturais exercem muita influência na vida das pessoas em suas comunidades. Que eles por terem o diagnóstico social e de saúde desta população poderão ter resultados relevantes como produto de suas pesquisas que terão impacto nestas sociedade, podendo facilitar as questões que permeiam a sexualidade diminuindo as diferenças e distância entre as pessoas.

\section{Conhecimentos dos enfermeiros sobre sexualidade no climatério}

Quanto ao conhecimento relacionado à sexualidade no climatério, o escore médio foi de 2,67 (nível de conhecimento de 53,4\%), sendo o terceiro melhor escore geral de conhecimento. No entanto, é muito claro que para entender a sexualidade das mulheres que vivenciam o climatério, é preciso entender sobre as possíveis alterações anatomofisiológicas que a mulher pode ter na referida fase. Se o escore de nível de conhecimento sobre a política de atenção à saúde da mulher no climatério, assim como as questões relacionadas à fisiologia da fase não foram satisfatórios $(2,67)$, os resultados deste escore se contradizem com a literatura.

Pesquisas que falam da interferência da fase do climatério na sexualidade indicam que os profissionais que possuem conhecimento sobre as mudanças orgânicas e suas consequências sobre a sexualidade podem desmistificar os acontecimentos diminuindo ao máximo os tabus e ajudá-las neste momento, desfazendo episódios que danificaram emocionalmente e a enfraquece perante seu lugar na coletividade (Garcia \& Lisboa, 2012).

Segundo Lorenzi e Saciloto, (2006), o impacto causado pela síndrome climatérica sobre a sexualidade da mulher, não está relacionado apenas às alterações hormonais, pois fatores psicossociais e culturais referentes ao envelhecimento também podem influenciar. 
Todas as mudanças pelas quais a mulher passa, e todos os efeitos endócrinos, físicos, psíquicos e sociais, deixam mais evidente à relevância das intervenções e de todas as formas de cuidar que ajudam a mulher a passar por esta fase, o que exige conhecimento para deixar claro a esta mulher que ela vive uma fase que tem começo e fim, que mais da metade da vida dela será vivenciada após esta fase (Garcia \& Lisboa, 2012). Portanto o enfermeiro tem que entender bem o processo para ser membro ativo das orientações e intervenções necessárias para ajudar a mulher a passar por este momento como autora do seu autocuidado.

Segundo Cabral et al., (2012), a sintomatologia do climatério pode levar as mulheres que vivenciam esta fase a desenvolverem disfunções sexuais. Mas, os fatores sociodemográficos também têm forte influência neste surgimento.

O conceito de (Abdo, 2006) diz que as disfunções sexuais podem ser definidas ainda como a incapacidade de participar do ato sexual com satisfação, acometendo ambos os sexos, mas entre as mulheres as queixas sobre a qualidade subjetiva da experiência sexual como um todo superam a falha de uma resposta restrita a um aspecto do ato sexual.

Que inúmeras causas podem prejudicar de forma pontual ou prolongada a resposta sexual da mulher, desencadeando as disfunções sexuais. A educação rígida, estimulação inadequada das zonas exógenas, conflitos conjugais, falta de atração pelo parceiro, história de violência sexual, ansiedade, depressão, fadiga, doenças físicas, coronariopatias, dislipidemias, diabetes, distúrbios hormonais. Assim como o uso de medicamentos diversos inibem a libido (Lopes \& Bousquat, 2011.

As disfunções sexuais se caracterizam por falta, excesso, desconforto e/ou dor no desenvolvimento do ciclo de resposta sexual, o que prejudica uma ou mais das fases deste ciclo (desejo, excitação e/ou orgasmo), podendo inclusive bloqueá-lo em determinado momento do seu desenvolvimento até a resolução (Lara et al., 2008). Como já citado no Brasil, 8,2 \% das mulheres se queixam de absoluta falta de desejo sexual; $26 \%$ têm dificuldade de excitação; $26,2 \%$ não atingem o orgasmo e $17,8 \%$ apresentam dispareunia (Lopes \& Bousquat, 2011).

$\mathrm{Na}$ atenção básica, o profissional enfermeiro realiza a consulta de enfermagem, e cuidar do enfermeiro neste momento tem foco na promoção à saúde e prevenção de doenças. A consulta voltada para a sexualidade humana segue à luz da educação em saúde. Desta forma, na ESF, a Enfermagem deve romper o silêncio, quando o que está em discussão é o lugar da sexualidade como componente essencial na integralidade do cuidado (Andrade, 2016; Cruz da Silva et al., 2013.).

A ESF é identificada como uma nova forma de promover a atenção, esta deve ser baseada na construção de vínculo entre os profissionais e usuários. O vínculo, cria uma referência profissional à comunidade, o possibilita que reconheça os profissionais, e estes se responsabilizam pela abordagem das necessidades de saúde, com a confiança, acolhimento, resolutividade e sigilo profissional (Andrade, 2016; Cruz da Silva et al., 2013.).

Diante dos fatos, podemos dizer que cuidar é o ato de facilitar, apoiar ou dar assistência ao indivíduo ou grupo, com a finalidade de melhorar a situação de vida das pessoas melhorando sua qualidade de vida e consequentemente promovendo à saúde (Zampieri et al., 2009).

E a sexualidade, estando inserida entre estas mudanças tão características do climatério, precisa ser inserida no contexto do cuidar, de orientar esta paciente visando o empoderamento sobre a situação e sobre as alternativas para vivenciar uma sexualidade mais assertiva e satisfatória (Cruz da Silva et al., 2013).

\section{Conclusão}

Existe a necessidade de transversalidade do cuidado de enfermagem à mulher no climatério desde o tratar do cuidado do corpo com propriedade e intimidade com o mesmo, seus aspectos mais elementares do cuidado com a higiene corporal até aqueles tecnicamente mais complexos, como identificar inadequações e disfunções sexuais prescrevendo os cuidados de enfermagem.

O que é fundamental, quando se faz promoção à saúde e educação em saúde na ESF, na saúde da mulher no climatério e especificamente na saúde sexual. Nessa perspectiva, a enfermagem precisa tratar a sexualidade como um dos pilares da 
qualidade de vida e conseguir aproximar-se desta mulher acolhendo e empoderando, tornando-se referência para a mesma e consequentemente mostrando que esta é mais uma fase da vida, e que com o cuidado devido à mulher passará com menos dificuldade e mais fortalecida.

Os treinamentos específicos também parecem não terem atingido os seus objetivos, e o conhecimento e a formação abaixo do desejado podem estar influenciando negativamente em sua prática. Portanto, o presente estudo revelou não só a insuficiência do aporte teórico, como também a deficiência das informações disseminadas nas capacitações e pós-graduações. Pois, diante de um conhecimento insuficiente desses profissionais, tanto o reconhecimento do início dessa fase quanto às devidas orientações poderá estar comprometido.

Assim, se faz necessário a garantia não apenas da realização de capacitações, mas que essas aconteçam com qualidade, tendo por finalidade causar de fato um impacto, nas condições da assistência prestada a essa população.

Acreditamos que os achados apresentados e as reflexões realizadas podem contribuir para a melhoria da assistência prestada às mulheres no climatério pelos profissionais de enfermagem na Estratégia Saúde da Família da cidade do Recife.

\section{Referências}

Abdo, C. (2006). Elaboração e validação do quociente sexual, versão feminina: uma escala para avaliar a função sexual da mulher. Revista Brasileira de Medicina, 63(9), 477-482.

Alves, C. N. (2020). A mulher climatérica: reflexões sobre desejo sexual, beleza e feminilidade. Estudos em Sexualidade. 2 , 126.

Andrade, A. R. L. de. (2016). Conhecimento de enfermeiros da estratégia saúde da família sobre sexualidade no climatério. (Dissertação de mestrado) Universidade de Pernambuco. Recife, PE, Brasil.

Aparecida, N., Alvim, T., De, M. \& Ferreira, A. (2007). Perspectiva problematizadora da educação popular em saúde e a enfermagem. Texto \& Contexto Enfermagem, 16 (2).

Brasil Ministério da saúde. (2008). Manual de Atenção à Mulher no Climatério. Menopausa. https://bvsms.saude.gov.br/bvs/publicacoes/manual_atencao_mulher_climaterio.pdf

Brasil. Ministério da Saúde. (2013). Saúde sexual e saúde reprodutiva. Cadernos de Atenção Básica, $n$. 26. https://bvsms.saude.gov.br/bvs/publicacoes/saude_sexual_saude_reprodutiva.pdf

Brasil. Ministério da Saúde. (2012). Resolução $\quad N^{\circ} \quad 466, \quad$ de $12 \quad$ de $\quad$ dezembro 2012 de https://bvsms.saude.gov.br/bvs/saudelegis/cns/2013/res0466_12_12_2012.html

Brasil. Ministério da Saúde. (1996). Resolução No 196, de 10 de outubro de 1996. https://www.mendeley.com/reference-manager/library/all-references/

Cabral, P. U. L. et al. (2012). Influência dos sintomas climatéricos sobre a função sexual de mulheres de meia-idade. Revista Brasileira de Ginecologia e Obstetrícia, 34(7), 329-334. https://doi.org/10.1590/S0100-72032012000700007

Canesqui, A. M., \& Spinelli, M. A. dos S. (2006). Saúde da família no Estado de Mato Grosso, Brasil: perfis e julgamentos dos médicos e enfermeiros. Cadernos de Saúde Pública, 22(9), 1881-1892. https://doi.org/10.1590/S0102-311X2006000900019

Carla, A. et al. (2010). Atuação do enfermeiro diante da importância da assistência à saúde da mulher no climatério the nurse actions regarding the importance of women healthcare in climacteric actitud del enfermero ante la importancia de la atención a la salud de la mujer en el climaterio. In remE-Rev. Min. Enferm $14(2)$.

Cestari, M. E. (2003). Padrões de conhecimento da enfermagem e suas implicações no ensino. https://www.seer.ufrgs.br/rgenf/article/view/4435

Cruz da Silva, S. et al. (2013). Análise da produção do conhecimento de enfermagem acerca da sexualidade dos adolescentes. Convibra Saúde.

De Souza, B. M. S. et al. (2021). Assistência à saúde da mulher climatérica: uma revisão de literatura. Research, Society and Development, 10(17), e26101724332-e26101724332. https://doi.org/10.33448/rsd-v10i17.24332

Lorenzi, D. R. S., \& Saciloto, B. (2006). Frequiência da atividade sexual em mulheres menopausadas. Revista Da Associação Médica Brasileira, 52(4), 256260. https://doi.org/10.1590/S0104-42302006000400027

Diniz Machado, M. E., Moreira Christoffel, M., \& de Souza Tassinari, W. (n.d.). Socio-demographic profiles and qualifications of neonatal nurses in developmental care: a descriptive study. http://www.objnursing.uff.br/index.php/nursing/article/view/4718

Fettermann, F. A. et al. (2021). Práticas do enfermeiro frente a sexualidade da mulher no climatério. In: Congresso Internacional em Saúde.

Ferrari, R. A. P., Thomson, Z., \& Melchior, R. (2005). Estratégia da saúde da família: perfil dos médicos e enfermeiros, Londrina, Paraná. Semina: Ciências Biológicas e Da Saúde, 26(2), 101. https://doi.org/10.5433/1679-0367.2005v26n2p101 
Fundação Oswaldo Cruz., \& Escorel, Sarah. (2005). Saúde da família: avaliação da implementação em dez grandes centros urbanos : síntese dos principais resultados. Editora MS.

Gd, S. et al. (2013). A sexualidade na formação acadêmica do enfermeiro. 17 (1).

Ito, E. E. et al. (2006). O ensino de enfermagem e as diretrizes curriculares nacionais: utopia x realidade. Revista Da Escola de Enfermagem Da USP, 40(4), 570-575. https://doi.org/10.1590/S0080-62342006000400017

Lara, L. A. da S. et al. (2008). Abordagem das disfunções sexuais femininas. Revista Brasileira de Ginecologia e Obstetrícia, 30(6), 312-321. https://doi.org/10.1590/S0100-72032008000600008

LAVÔR, R. M. de. (2010). Estratégia de saúde da família na assistência à mulher na fase do climatério. http://dspace.sti.ufcg.edu.br:8080/jspui/handle/riufcg/13229

Lima Gonçalves, R. et al. (2013). The experience of sexuality through the view of women during pregnancy a vivência da sexualidade na perspectiva de mulheres no período gestacional la experiencia de la sexualidad en vista de las mujeres durante la gestación. J Nurs UFPE on Line, 7(1), 196-204. https://doi.org/10.5205/reuol.3049-24704-1-LE.0701201327

Mendes Pinto Neto, Aa., Lúcia Ribeiro Valadares, A., Costa-Paiva, L. (2012). Climatério e sexualidade Climacteric and sexuality. https://doi.org/https://doi.org/10.1590/S0100-72032013000300001

Rocha, B. S. et al. (2009). Enfermeiros coordenadores de equipe do PSF. In Rev. enferm. UERJ. 17(2).

Silva, M. G., Dias, M. S., \& Oliveira, M. P. (2019). O período climatério sob ótica da mulher. Revista saber digital, 12(1), 29-38.

Sodson Da, A., \& Silva, R. (2009). Assistência realizada por enfermeiros do psf a mulher no climatério. Service held for nurse of PSF to climacteric women. Ano IV, 1.

Valença, C. N., Nascimento Filho, J. M. do, \& Germano, R. M. (2010). Mulher no climatério: reflexões sobre desejo sexual, beleza e feminilidade. Saúde e Sociedade, 19(2), 273-285. https://doi.org/10.1590/S0104-12902010000200005

Zampieri, M. de F. M. et al. (2009). O processo de viver e ser saudável das mulheres no climatério. Escola Anna Nery, 13(2), 305-312. https://doi.org/10.1590/S1414-81452009000200010

Zanetti, T. G. et al. (2010). Perfil socioprofissional e formação de profissionais de equipes de saúde da família: um estudo de caso. Ciência, Cuidado e Saúde, 9(3). https://doi.org/10.4025/cienccuidsaude.v9i3.7664 